\title{
ON THE DIRECT BEM FORMULATION IN THE DUAL SYSTEM OF PLANE ELASTICITY FOR ORTHOTROPIC BODIES
}

\author{
JUDIT DUDRA \\ Institute of Logistics and Production Systems \\ H-3519 Miskolctapolca, Iglói út 2. Hungary \\ judit.dudra@gmail.com \\ GYÖRGY SZEIDL \\ Institute of Applied Mechanics, University of Miskolc \\ H-3515 Miskolc, Miskolc-Egyetemváros, Hungary \\ gyorgy.szeidl@uni-miskolc.hu
}

[Received: February 28, 2015, Accepted: September 18, 2015.]

Dedicated to Professor Barna Szabó on the occasion of his eightieth birthday and to Professor Imre Kozák on the occasion of his eighty-fifth birthday

\begin{abstract}
The paper is devoted to plane problems of orthotropic bodies in a dual formulation. After presenting the governing equations in terms of stress functions of order one, we determine the two fundamental solutions and set up the dual Somigliana relations both for inner and exterior regions. These include the boundary integral equations of the direct method. A constant stress state at infinity is part of the formulation established for exterior regions. We also derive an integral representation of the stresses. The numerical examples presented illustrate the applicability of the direct boundary integral equations.
\end{abstract}

Mathematical Subject Classification: 74S15, 45F15

Keywords: Plane problems, orthotropic bodies, stress functions of order one, the dual Somigliana formulae, direct boundary element method

\section{INTRODUCTION}

According to the famous TONTI scheme [1, in the primal system of elasticity the displacement field is the basic variable while the strains and stresses are the intermediate variables of the first and second kind. Body forces are referred to as the source variable. Problems in the primal system are governed by the primal kinematic equations, which relate the strains to the displacements; HookE's law, which connects the primal intermediate variables of the second kind to those of the first kind; and the equilibrium or primal balance equations, written in terms of the intermediate variables of the second kind. 
In the dual system of elasticity, stress functions are the basic variables, and stresses and strains constitute the intermediate variables of the first and second kind. A prescribed incompatibility, which is in general zero, is the source variable. Problems in the dual system are governed by the dual kinematic equations, which express the stresses in terms of stress functions; the inverse form of HookE's law, which relates the dual intermediate variables of the second kind to that of the first kind; and the compatibility equations (dual balance equations).

In a classical paper, RIzzo and SHIPPy [2] solve plane problems by the direct boundary element method for which physical quantities (in the primal system the displacements and the stress vector) are the unknowns on the boundary. These authors assume that the body is orthotropic and focus on an inner region. The most important relations are presented for anisotropic bodies as well. As in earlier work by RIzzo [3], the numerical solution is based on a constant approximation of the displacements and the stress vector over boundary elements.

A number of subsequent papers study the boundary value problems of plane elasticity using the boundary element method under the assumption that the material is orthotropic or anisotropic. VABLE and SIKARSKIE apply the indirect method for which the solution is sought in terms of appropriately chosen potential functions 4 . SHIAH and TAN transform 2D and 3D anisotropic field problems in such a way that the Laplace operator becomes the operator of the basic equation, a procedure known as direct domain mapping [5, 6]. However, these results can only be applied to problems of elasticity when it is possible to define a displacement potential which satisfies the basic equation investigated by Shiah and TAN. Hung, SAn, LiU and Zen [7] also study orthotropic bodies and provide additional references. We emphasize that all these papers [2, 3, 4, 7] as well as the books [8, 9] and the references they cite use the primal system of plane elasticity.

While many papers study plane problems in the primal system, there are only a few which use the dual system and treat the real stress functions of order one as the basic variables. One advantage of using stress functions of order one is that calculating stresses only requires determining first derivatives, while with stress functions of order two the stresses are obtained in terms of the second derivatives. First derivatives are more convenient in boundary element applications, although an additional equation is needed to ensure that the stresses are symmetric. Assuming homogenous and isotropic materials and using stress functions of order one, SzEIDL investigated the plane problem with the direct boundary element method in the dual system of elasticity [10, 11].

Our goal in this paper is to extend the results presented in [10, 11] as well as those in the thesis 12 for homogenous but orthotropic materials. We develop the fundamental solutions and the Somigliana relations in the dual system of plane elasticity for an orthotropic body provided that the stress functions of order one are the basic variables. We then set up a direct boundary element formulation and present an algorithm for numerical solutions. The examples shown illustrate the applicability of the algorithm. 
The paper is organized into eight sections. Section 2 presents the governing equation of the problem in a dual formulation. The fundamental solutions of order one and two are determined in Section 3. The dual Somigliana relations for inner and outer regions are derived in Sections 4 and 5. Section 6 deals with the determination of the stresses on the boundary. The last two sections present two numerical examples and a conclusion.

\section{Governing EQUATIOnS}

Throughout this paper $x_{1}=x$ and $x_{2}=y$ are rectangular Cartesian coordinates, referred to an origin $O$. For vectorial and tensorial quantities indicial notations are used. \{Greek\}[Latin subscripts] are assumed to have the range $\{(1,2)\}[(1,2,3)]$, summation over repeated indices is implied, $\delta_{\kappa \lambda}$ is the Kronecker delta, $\varepsilon_{p q r}$ is the permutation symbol. If an index in a summation is repeated more than twice, we also typeset the summation symbol to avoid misunderstanding (see for instance equation (3.9) below).

The inner and outer regions shown in Figure 1 are denoted by $A_{i}$ and $A_{e}$. They are bounded by the contour

$$
\mathcal{L}_{0}=\mathcal{L}_{t 1} \cup \mathcal{L}_{u 2} \cup \mathcal{L}_{t 3} \cup \mathcal{L}_{u 4} .
$$

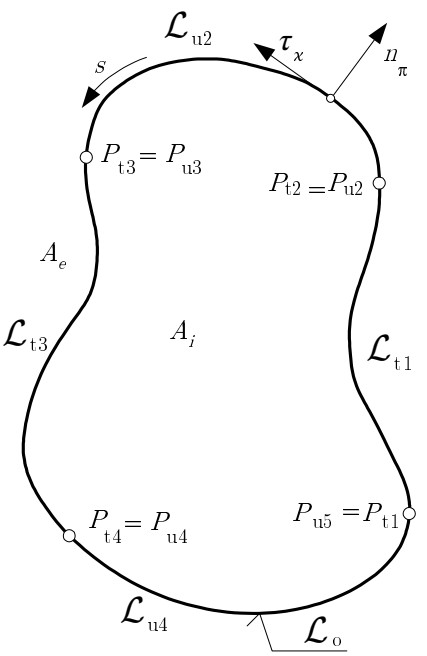

Figure 1.

We assume that [displacements] $\{$ tractions $\}$ are imposed on the arc $\left[\mathcal{L}_{u}=\mathcal{L}_{u 2} \cup\right.$ $\left.\mathcal{L}_{u 4}\right]\left\{\mathcal{L}_{t}=\mathcal{L}_{t 1} \cup \mathcal{L}_{t 3}\right\}$. We stipulate that the contour has a unit tangent $\tau_{\kappa}$ and admits an appropriate parametrization in terms of its arc length $s$. The outer unit normal is denoted by $n_{\pi}$. In accordance with the notations introduced $\partial_{\alpha}$ stands for the derivatives taken with respect to $x_{\alpha}$. Assuming plane problems let $u_{\kappa}, e_{\kappa \lambda}$ and $t_{\kappa \lambda}$ be the displacement field and the in plane components of strain and stress, respectively. The stress functions of order one are denoted by $\mathcal{F}_{\rho}$.

We shall assume that there are no body forces.

For homogenous and orthotropic material the plane problem of classical elasticity in the dual system of elasticity is governed by

1. the dual kinematic equations

$$
\begin{array}{cl}
t_{11}=\mathcal{F}_{1} \partial_{2}, & t_{12}=\mathcal{F}_{2} \partial_{2}, \\
t_{21}=-\mathcal{F}_{1} \partial_{1}, & t_{22}=-\mathcal{F}_{2} \partial_{1},
\end{array}
$$


which express the stresses in terms of stress functions of order one,

2. the inverse form of HookE's law

$$
\begin{gathered}
e_{11}=u_{1} \partial_{1}=s_{11} t_{11}+s_{12} t_{22}, \quad e_{22}=u_{2} \partial_{2}=s_{21} t_{11}+s_{22} t_{22}, \\
e_{12}=\frac{1}{2}\left(u_{1} \partial_{2}+u_{2} \partial_{1}\right)=\frac{s_{66}}{4}\left(t_{12}+t_{21}\right) ;
\end{gathered}
$$

where $s_{11}, s_{12}, s_{21}$ and $s_{22}$ stand for the constants of elasticity ${ }^{1}$

3. the compatibility conditions

$$
e_{11} \partial_{2}-e_{12} \partial_{1}+\varphi_{3} \partial_{1}=0, \quad e_{21} \partial_{2}-e_{22} \partial_{1}+\varphi_{3} \partial_{2}=0
$$

in which $\varphi_{3}$ is the rotation field

4. and the symmetry condition

$$
t_{12}=t_{21} \text {. }
$$

If this equation is fulfilled then either equation $(2.1 \mathrm{a})_{2}$ or equation $(2.1 \mathrm{~b})_{1}$ can be omitted. Thus we have nine equations for the nine unknowns $\mathcal{F}_{1}, \mathcal{F}_{2}, t_{11}$, $t_{12}=t_{21}, t_{22}, e_{11}, e_{12}=e_{21}, e_{22}$ and $\varphi_{3}$.

Field equations (2.1a b), 2.2a, b), 2.3 and (2.4) should be associated with appropriate boundary conditions. If the contour is not divided into parts then either tractions or displacements can be imposed on it. In the opposite case the contour is assumed to be divided into arcs of even number on which displacements and tractions are imposed alternately. As mentioned earlier, in Figure 1 \{tractions\}[displacements] are prescribed on the arc $\left\{\mathcal{L}_{t}\right\}\left[\mathcal{L}_{u}\right]$. Variables with hats stand for the values prescribed: $\hat{u}_{\kappa}, \hat{t}_{\rho}$ and $\hat{\mathcal{F}}_{\rho}$ are the prescribed displacements, tractions (stress vector) and stress functions, respectively.

For the sake of a formal similarity of the boundary integral equations regarded in primal and dual formulations we introduce the notation

$$
\mathfrak{t}_{\lambda}=-n_{\kappa}\left(\varepsilon_{\kappa \rho 3} e_{\rho \lambda}-\delta_{\kappa \lambda} \varphi_{3}\right) \quad s \in \mathcal{L}_{o},
$$

where on the other hand

$$
\mathfrak{t}_{\lambda}=-\frac{\mathrm{d} u_{\kappa}}{\mathrm{d} s} \quad s \in \mathcal{L}_{o} .
$$

We refer to $\mathfrak{t}_{\lambda}$ as the dual stress vector and to its elements as dual stresses. Observe that the same letter denotes both the stresses and the dual stresses; we distinguish between them by typesetting the dual stresses in calligraphic fonts. The same notational convention will be used for the dual displacements defined by equation $(3.2)_{2}$.

The dual field equations $2.1 \mathrm{a}, \ldots, 2.4$ should be associated with the strain boundary conditions of the form

$$
\mathfrak{t}_{\kappa}=-\frac{\mathrm{d} \hat{u}_{\kappa}}{\mathrm{d} s} \quad s \in \mathcal{L}_{u}
$$

\footnotetext{
${ }^{1}$ The strain energy density $u$ should be strictly positive: $2 u=t_{11}\left(s_{11} t_{11}+s_{12} t_{22}\right)+t_{22}\left(s_{21} t_{11}+s_{22} t_{22}\right)+\frac{s_{66}}{4}\left(t_{12}+t_{21}\right)\left(t_{12}+t_{21}\right)>0$.
} 
(note that one can not prescribe boundary conditions directly on the displacements since they do not belong to the set of dual variables) and a set of boundary conditions imposed on the stress functions

$$
\mathcal{F}_{\rho}(s)-\underbrace{\mathcal{F}_{\rho}\left(P_{t i}\right)}_{(t i)^{\rho}}=\underbrace{\int_{P_{t i}}^{s} \hat{t}_{\rho}(\sigma) d \sigma}_{\hat{\mathcal{F}}_{\rho}(s)}, \quad s \in \mathcal{L}_{t i}, \quad i=1,3
$$

where the integral with value $\hat{\mathcal{F}}_{\rho}(s)$ on the right side is the resultant of the tractions prescribed on the arc $\left[P_{t i}, s\right]$ while $\underset{(t i)}{C} \rho$ is an undetermined constant of integration.

The supplementary conditions of single valuedness can easily be obtained from equation (2.6). These conditions express displacement continuity at the endpoints of the $\operatorname{arcs} \mathcal{L}_{t i}$ regarded on the contour and have the form

$$
\int_{\mathcal{L}_{t i}} n_{\kappa}\left(\varepsilon_{\kappa \rho 3} e_{\rho \lambda}-\delta_{\kappa \lambda} \varphi_{3}\right) \mathrm{d} s-\left.\hat{u}_{\lambda}\right|_{P_{t i}} ^{P_{t, i+1}}=0 \quad(i=1,3) .
$$

Observe that we have as many undetermined constants of integration as there are supplementary conditions of single valuedness.

One undetermined constant of integration $\underset{(t i)}{C_{\rho} \rho}$ can be set to zero without loss of generality, since the equations $2.1 \mathrm{~b}$ c) contain only the derivatives of the stress functions. It can also be shown that the supplementary conditions of single valuedness 2.8 are not independent, i.e., one condition can always be omitted. A proof for the latter statement assuming isotropic bodies and plane problems can be found in the thesis [13] by Szeidl.

\section{BASIC EQUATION AND FUNDAMENTAL SOLUTIONS}

After eliminating the intermediate variables $e_{\kappa \lambda}$ and $t_{\kappa \lambda}$ from the compatibility conditions $(2.3)$ and the symmetry condition 2.4 we obtain the basic equation in the form

$$
\mathfrak{D}_{i k} \mathfrak{u}_{k}=0 \quad i=1,2,3
$$

where $\mathfrak{D}_{i k}$ is a differential operator and $\mathfrak{u}_{k}$ is the vector of fundamental variables (the stress functions $\mathfrak{u}_{\kappa}$ - see below - will be referred to as dual displacements):

$$
\left[\mathfrak{D}_{i k}\right]=\left[\begin{array}{ccc}
s_{11} \partial_{2} \partial_{2}+\frac{s_{66}}{4} \partial_{1} \partial_{1} & -\left(s_{12}+\frac{s_{66}}{4}\right) \partial_{1} \partial_{2} & -\partial_{1} \\
-\left(s_{21}+\frac{s_{66}}{4}\right) \partial_{1} \partial_{2} & s_{22} \partial_{1} \partial_{1}+\frac{s_{66}}{4} \partial_{2} \partial_{2} & -\partial_{2} \\
-\partial_{1} & -\partial_{2} & 0
\end{array}\right], \quad \mathfrak{u}_{k}=(\underbrace{\mathcal{F}_{1}, \mathcal{F}_{2}}_{\mathfrak{u}_{\kappa}},-\varphi_{3}) .
$$

Let $Q\left(\xi_{1}, \xi_{2}\right)$ and $M\left(x_{1}, x_{2}\right)$ be two points in the plane of strain: the source point and the field point. We shall assume temporarily that the point $Q$ is fixed. The distance between $Q$ and $M$ is $R$, the position vector of $M$ relative to $Q$ is $r_{\kappa}$. We refer to the 
solution of the differential equation

$$
\stackrel{M}{\mathfrak{D}}_{i k} \mathfrak{u}_{k}+\delta(M-Q) e_{i}(Q)=0, \quad i=1,2,3
$$

as the fundamental solution. Here $\delta(M-Q)$ stands for the Dirac function, $e_{\kappa}(Q)$ is a prescribed incompatibility and $e_{3}(Q)$ is a couple perpendicular to the plane of strain. We determine the fundamental solution following the approach of KUPRADZE [14, here we only present the main steps in the derivation.

Let $D_{k l}$ be the cofactor of $\mathfrak{D}_{i k}$

$$
\mathfrak{D}_{i k} D_{k l}=\operatorname{det}\left(\mathfrak{D}_{m n}\right) \delta_{i l}
$$

We seek the fundamental variables $\mathfrak{u}_{k}$ in the form

$$
\mathfrak{u}_{k}=D_{k l} \chi_{l},
$$

where $\chi_{l}=\chi e_{l}(Q)$ and $\chi$ is the Galjorkin function. If we substitute 3.5 into 3.3 and take (3.4) into account we get

$$
\operatorname{det}\left(\mathfrak{D}_{m n}\right) \chi+\delta(M-Q)=0
$$

where

$$
\operatorname{det}\left(\mathfrak{D}_{m n}\right)=-\left[s_{11} \partial_{2}^{4}+\left(2 s_{21}+s_{66}\right) \partial_{1}^{2} \partial_{2}^{2}+s_{22} \partial_{1}^{4}\right]
$$

For the sake of later calculations we introduce the following quantities

$$
\begin{gathered}
\alpha_{1,2}^{2}=-\frac{2 s_{21}+s_{66}}{2 s_{11}} \pm \sqrt{\left(\frac{2 s_{21}+s_{66}}{2 s_{11}}\right)^{2}-\frac{s_{22}}{s_{11}}}, \quad \beta_{\kappa}^{2}=-1 / \alpha_{\kappa} \\
\rho_{\kappa}=\left(x_{1}-\xi_{1}\right)+\beta_{\kappa}\left(x_{2}-\xi_{2}\right), \\
d_{1}=-\left|\begin{array}{ccc}
1 & \tilde{\beta}_{1} & \tilde{\beta}_{1}^{2} \\
1 & \beta_{2} & \beta_{2}^{2} \\
1 & \tilde{\beta}_{2} & \tilde{\beta}_{2}^{2}
\end{array}\right|, d_{2}=-\left|\begin{array}{ccc}
1 & \beta_{1} & \beta_{1}^{2} \\
1 & \tilde{\beta}_{1} & \tilde{\beta}_{1}^{2} \\
1 & \tilde{\beta}_{2} & \tilde{\beta}_{2}^{2}
\end{array}\right|,
\end{gathered}
$$

$\left(\tilde{\beta}_{\kappa}\right.$ is the complex conjugate of $\left.\beta_{\kappa}\right)$

$$
\begin{gathered}
\mathcal{K}=-\frac{1}{I_{1}+I_{2}}, \quad I_{\kappa}=4 \pi \frac{d_{\kappa}}{b_{\kappa}+1}\left(b_{\kappa}^{3} s_{11}+b_{\kappa}^{2}\left(s_{21}+s_{66}\right)-b_{\kappa} s_{12}-s_{22}\right), \\
b_{1}=\sqrt{D_{1}-\sqrt{D}}, \quad b_{2}=\sqrt{D_{1}+\sqrt{D}} \\
D=\left(\frac{2 s_{21}+s_{66}}{2 s_{11}}\right)^{2}-\frac{s_{22}}{s_{11}}, \quad D_{1}=\frac{2 s_{21}+s_{66}}{2 s_{11}} \\
a_{33}=s_{11} s_{22}+\left(\frac{s_{66}}{4}\right)^{2}-\left(s_{21}+\frac{s_{66}}{4}\right)^{2} .
\end{gathered}
$$
form

Using the quantities introduced it can be shown that the solution for $\chi$ takes the

$$
\chi(M, Q)=\mathcal{K} \operatorname{Im} \sum_{\kappa=1}^{2} d_{\kappa} \rho_{\kappa}^{2} \ln \rho_{\kappa} .
$$


With the GaLERKIN function $\chi$ it follows from equation 3.5 that

$$
\mathfrak{u}_{k}=D_{k l} \chi_{l}=\underbrace{D_{k l} \chi}_{\mathfrak{U}_{k l}} e_{l}=\mathfrak{U}_{k l}(M, Q) e_{l}(Q),
$$

where $\mathfrak{U}_{k l}(M, Q)$ is the matrix of the fundamental solution of order one. Omitting the long formal transformations we find that

$$
\begin{aligned}
& {\left[\mathfrak{U}_{p q}(Q, M)\right]=\operatorname{Im} \sum_{k=1}^{2} \mathcal{K} \times} \\
& \times\left[\begin{array}{ccc}
d_{k}\left(2 \ln \rho_{k}+3\right) \beta_{k}^{2} & d_{k}\left(2 \ln \rho_{k}+3\right) \beta_{k} & \frac{2 d_{k}}{\rho_{k}}\left[-\left(s_{21}+\frac{s_{66}}{2}\right) \beta_{k}^{2}+s_{22}\right] \\
d_{k}\left(2 \ln \rho_{k}+3\right) \beta_{k} & d_{k}\left(2 \ln \rho_{k}+3\right) & \frac{2 d_{k} \beta_{k}}{\rho_{k}}\left[\left(s_{12}+\frac{s_{66}}{2}\right)-s_{11} \beta_{k}^{2}\right] \\
\frac{2 d_{k}}{\rho_{k}}\left[s_{22}-\left(s_{21}+\frac{s_{66}}{2}\right) \beta_{k}^{2}\right] & \frac{2 d_{k} \beta_{k}}{\rho_{k}}\left[\left(s_{21}+\frac{s_{66}}{2}\right)-s_{11} \beta_{k}^{2}\right] & \left\{s_{21} s_{66}+\frac{s_{66}^{2}}{2}-2 a_{33}\right\} \beta_{k}^{2} \frac{d_{k}}{\rho_{k}^{2}}
\end{array}\right]
\end{aligned}
$$

Recalling the dual kinematic equations $2.1 \mathrm{a}$ b) we obtain the fundamental solutions for the stresses $t_{11}, t_{12}=t_{21}$ and $t_{22}$ by calculating from the following equations:

$$
\begin{aligned}
& t_{11}=\mathcal{F}_{1} \stackrel{M}{\partial}{ }_{2}=\stackrel{M}{\stackrel{M}{\partial}} \mathfrak{u}_{2}=\stackrel{M}{\partial}{ }_{2} \mathfrak{U}_{1 l}(M, Q) e_{l}(Q), \\
& t_{12}=\mathcal{F}_{2} \stackrel{M}{\partial}{ }_{2}=\stackrel{M}{\partial}{ }_{2} \mathfrak{U}_{2 l}(M, Q) e_{l}(Q)=t_{21}=-\mathcal{F}_{1} \stackrel{M}{\partial}{ }_{1}=-\stackrel{M}{\partial} \mathfrak{U}_{1 l}(M, Q) e_{l}(Q), \\
& t_{22}=-\mathcal{F}_{2} \stackrel{M}{\partial}=-\stackrel{M}{{ }_{1}}=\stackrel{M}{\partial} \mathfrak{u}_{2} \stackrel{\mathfrak{U}}{2 l}_{1}(M, Q) e_{l}(Q) .
\end{aligned}
$$

After performing the necessary calculations we have

$$
\left[\begin{array}{c}
t_{11} \\
t_{12} \\
t_{22}
\end{array}\right]=\mathcal{K} \operatorname{Im} \sum_{k=1}^{2} \frac{2 d_{k}}{\rho_{k}}\left[\begin{array}{ccc}
-\beta_{k}^{3} & \beta_{k}^{2} & -\frac{\beta_{k}}{\rho_{k}}\left[\left(s_{21}+\frac{s_{66}}{2}\right) \beta_{k}^{2}+s_{22}\right] \\
\beta_{k}^{2} & -\beta_{k} & \frac{1}{\rho_{k}}\left[\left(s_{21}+\frac{s_{66}}{2}\right) \beta_{k}^{2}+s_{22}\right] \\
-\beta_{k} & 1 & \frac{\beta_{k}}{\rho_{k}}\left[\left(s_{12}+\frac{s_{66}}{2}\right)+s_{11} \beta_{k}^{2}\right.
\end{array}\right]\left[\begin{array}{l}
e_{1} \\
e_{2} \\
e_{3}
\end{array}\right] .
$$

With the knowledge of the stresses, the strains can be obtained from the Hooke law (2.2a, b):

$$
\begin{aligned}
& {\left[\begin{array}{l}
e_{11} \\
e_{12} \\
e_{22}
\end{array}\right]=\mathcal{K} \operatorname{Im} \sum_{k=1}^{2} \frac{2 d_{k}}{\rho_{k}} \times} \\
& \times\left[\begin{array}{ccc}
-s_{11} \beta_{k}^{3}-s_{12} \beta_{k} & s_{11} \beta_{k}^{2}+s_{12} & -\frac{\beta_{k}}{\rho_{k}}\left[\left(s_{12}+\frac{s_{66}}{2}\right)\left(s_{11} \beta_{k}^{2}-s_{12}\right)+s_{11}\left(s_{22}-s_{12} \beta_{k}^{2}\right)\right] \\
\frac{1}{2} s_{66} \beta_{k}^{2} & -\frac{1}{2} s_{66} \beta_{k} & \frac{s_{66}}{2 \rho_{k}}\left[\left(s_{21}+\frac{s_{66}}{2}\right) \beta_{k}^{2}+s_{22}\right] \\
-s_{21} \beta_{k}^{3}-s_{22} \beta_{k} & s_{21} \beta_{k}^{2}+s_{22} & -\frac{\beta_{k}}{\rho_{k}}\left[\left(s_{21}+\frac{s_{66}}{2}\right)\left(s_{21} \beta_{k}^{2}-s_{22}\right)+s_{22}\left(s_{21}-s_{11} \beta_{k}^{2}\right)\right]
\end{array}\right]\left[\begin{array}{l}
e_{1} \\
e_{2} \\
e_{3}
\end{array}\right] \text {. }
\end{aligned}
$$


The dual stress vector is defined by equation (2.5a). We can compute the dual stress vector from the fundamental solution of order one to obtain

$$
\mathfrak{t}_{\kappa}=-\frac{\mathrm{d} u_{\kappa}}{d s}=e_{l}(Q) \mathcal{T}_{l \kappa}(\stackrel{o}{M}, Q)
$$

where $\mathcal{T}_{l \kappa}$ is referred to as fundamental solution of order two. The formal calculations are based on equation (2.5a b), which can be rewritten in a matrix form:

$$
-\frac{\mathrm{d}}{\mathrm{d} s}\left[\begin{array}{l}
u_{1} \\
u_{2}
\end{array}\right]=\left[\begin{array}{ccc}
n_{2} & -n_{1} & 0 \\
0 & n_{2} & -n_{1}
\end{array}\right]\left[\begin{array}{l}
e_{11} \\
e_{12} \\
e_{22}
\end{array}\right]+\left[\begin{array}{l}
n_{1} \\
n_{2}
\end{array}\right] \varphi_{3}
$$

We remark that these calculations require the use of equation $(3.16)$ and $e_{l}(Q) \mathfrak{U}_{l 3}\left(M_{o}, Q\right)$ - the latter provides $\varphi_{3}$. Omitting the details, we obtain for $\mathcal{T}_{l \kappa}$ :

$$
\begin{aligned}
\mathfrak{T}_{11}\left(M_{o}, Q\right)=\mathcal{K} \operatorname{Im} \sum_{\kappa=1}^{2} \frac{2 d_{\kappa}}{\rho_{\kappa}}\left\{n _ { 2 } \left(-s_{11} \beta_{\kappa}^{3}-\right.\right. & \left.s_{12} \beta_{\kappa}\right)-\frac{n_{1}}{2} s_{66} \beta_{\kappa}^{2}- \\
& \left.-n_{1}\left[s_{22}+\left(s_{21}+\frac{s_{66}}{2}\right) \beta_{\kappa}^{2}\right]\right\},
\end{aligned}
$$

$$
\begin{aligned}
\mathfrak{T}_{21}\left(M_{o}, Q\right)=\mathcal{K} \operatorname{Im} \sum_{\kappa=1}^{2} \frac{2 d_{\kappa}}{\rho_{\kappa}}\left\{n _ { 2 } \left(s_{11} \beta_{\kappa}^{2}+\right.\right. & \left.s_{12}\right)+\frac{n_{1}}{2} s_{66} \beta_{\kappa}- \\
& \left.-n_{1} \beta_{\kappa}\left[\left(s_{12}+\frac{s_{66}}{2}\right)+s_{11} \beta_{\kappa}^{2}\right]\right\},
\end{aligned}
$$

$$
\begin{aligned}
\mathfrak{T}_{12}\left(M_{o}, Q\right)=\mathcal{K} \operatorname{Im} \sum_{\kappa=1}^{2} \frac{2 d_{\kappa}}{\rho_{\kappa}}\left\{\frac{n_{2}}{2} s_{66} \beta_{\kappa}^{2}+n_{1}\left(s_{21} \beta_{\kappa}^{3}+s_{22} \beta_{\kappa}\right)-\right. & \\
& \left.-n_{2}\left[s_{22}+\left(s_{21}+\frac{s_{66}}{2}\right) \beta_{\kappa}^{2}\right]\right\},
\end{aligned}
$$

$$
\begin{aligned}
\mathfrak{T}_{22}\left(M_{o}, Q\right)=\mathcal{K} \operatorname{Im} \sum_{\kappa=1}^{2} \frac{2 d_{\kappa}}{\rho_{\kappa}}\left\{-\frac{n_{2}}{2} s_{66} \beta_{\kappa}\right. & -n_{1}\left(s_{21} \beta_{\kappa}^{2}+s_{22}\right)- \\
& \left.-n_{2} \beta_{\kappa}\left[\left(s_{12}+\frac{s_{66}}{2}\right)+s_{11} \beta_{\kappa}^{2}\right]\right\}
\end{aligned}
$$

$$
\begin{array}{r}
\mathfrak{T}_{31}\left(M_{o}, Q\right)=\mathcal{K} \operatorname{Im} \sum_{\kappa=1}^{2} \frac{2 d_{\kappa}}{\rho_{\kappa}}\left\{-\frac{n_{2} \beta_{\kappa}}{\rho_{\kappa}}\left[\left(s_{12}+\frac{s_{66}}{2}\right)\left(s_{11} \beta_{\kappa}^{2}-s_{12}\right)+s_{11}\left(s_{22}-s_{12} \beta_{\kappa}^{2}\right)\right]-\right. \\
\left.-\frac{n_{1} s_{66}}{2 \rho_{\kappa}}\left[\left(s_{21}+\frac{s_{66}}{2}\right) \beta_{\kappa}^{2}+s_{22}\right]+n_{1} \frac{\beta_{\kappa}^{2}}{\rho_{\kappa}}\left[a_{33}-\frac{s_{21} s_{66}}{2}-\frac{s_{66}^{2}}{4}\right]\right\}, \quad(3.19 \mathrm{e})
\end{array}
$$

$$
\begin{aligned}
\mathfrak{T}_{32}\left(M_{o}, Q\right) & =\mathcal{K} \operatorname{Im} \sum_{\kappa=1}^{2} \frac{2 d_{\kappa}}{\rho_{\kappa}}\left\{\frac{n_{2} s_{66}}{2 \rho_{\kappa}}\left[\left(s_{21}+\frac{s_{66}}{2}\right) \beta_{\kappa}^{2}+s_{22}\right]+\right. \\
& +\frac{n_{1} \beta_{\kappa}}{\rho_{\kappa}}\left[\left(\left(s_{21}+\frac{s_{66}}{2}\right)\left(s_{21} \beta_{\kappa}^{2}-s_{22}\right)\right)+s_{22}\left(s_{21}-s_{11} \beta_{\kappa}^{2}\right)\right]+
\end{aligned}
$$




$$
\left.+n_{2} \frac{\beta_{\kappa}^{2}}{\rho_{\kappa}}\left[a_{33}-\frac{s_{21} s_{66}}{2}-\frac{s_{66}^{2}}{4}\right]\right\} .
$$

It can also be shown by long hand made calculations that every column of the fundamental solutions $\mathfrak{U}_{k l}$ and $\mathfrak{T}_{k \lambda}$ satisfies the basic equations if $Q \neq M$.

\section{Dual Somigliana formulae for inner Regions}

The functions $\mathcal{F}_{\psi}, t_{\kappa \lambda}, e_{\kappa \lambda}$ and $\varphi_{3}$ are called an elastic state of the region $A_{i}$ if they satisfy the corresponding field equations. Let

$$
\mathcal{F}_{\psi}, t_{\kappa \lambda}, e_{\kappa \lambda}, \varphi_{3} \quad \text { and } \quad \stackrel{*}{\mathcal{F}}_{\psi}, \stackrel{*}{t}_{\kappa \lambda}, \stackrel{*}{e}_{\kappa \lambda}, \stackrel{*}{\varphi}_{3}
$$

be two elastic states of the region $A_{i}$. Integrating by parts, one can show that the relation

$$
\begin{aligned}
& \int_{A_{i}} \underbrace{\mathcal{F}_{\lambda}\left[\epsilon_{\kappa \rho 3}{ }^{*} e_{\kappa \lambda} \partial_{\rho}+\stackrel{*}{\varphi}_{3} \partial_{\lambda}\right]}_{\mathfrak{u}_{\lambda}\left(\mathfrak{D}_{\lambda l} \mathfrak{u}_{l}\right)} d A-\int_{A_{i}} \underbrace{\varphi_{3}\left(\stackrel{*}{\mathcal{F}}_{\psi} \partial_{\psi}\right)}_{-\mathfrak{u}_{3} \mathfrak{D}_{3 l} \mathfrak{u}_{l}} d A- \\
& -\int_{A_{i}}\left[\epsilon_{\kappa \rho 3} e_{\kappa \lambda} \partial_{\rho}+\varphi_{3} \partial_{\lambda}\right] \stackrel{*}{\mathcal{F}}{ }_{\lambda} d A+\int_{A_{i}}\left(\mathcal{F}_{\psi} \partial_{\psi}\right) \stackrel{*}{\varphi}_{3} d A=
\end{aligned}
$$

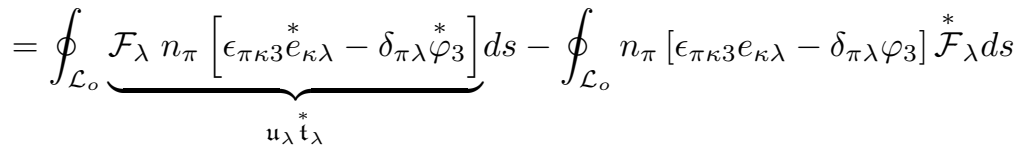

is an identity, referred to as the dual Somigliana identity. We can also write this identity in a more concise form

$$
\int_{A}\left[\mathfrak{u}_{r}\left(\mathfrak{D}_{r l}{ }^{*} \mathfrak{u}_{l}\right)-\stackrel{*}{u}_{r}\left(\mathfrak{D}_{r l} \mathfrak{u}_{l}\right)\right] d A=\oint_{\mathcal{L}_{o}}\left[\mathfrak{u}_{\lambda}^{*} \mathfrak{t}_{\lambda}-\mathfrak{u}_{\lambda}^{*} \mathfrak{t}_{\lambda}\right] d s
$$

Henceforward let $\mathfrak{u}_{l}(M)$ be an elastic state of the region $A_{i}$. Suppose that the other elastic state, denoted by ${ }^{*}$, is the one which belongs to the fundamental solutions:

$$
\stackrel{*}{\mathfrak{u}}_{l}(M)=e_{k}(Q) \mathfrak{U}_{k l}(M, Q)
$$

The latter is singular at the point $Q$. Consequently depending on the position of point $Q$ relative to the region $A_{i}$ we distinguish three cases - two of them are shown in Figure 2.

1. If $Q \in A_{i}$, then the neighborhood of $Q$ with radius $R_{\varepsilon}$, which is denoted by $A_{\varepsilon}$ and is assumed to lie wholly in $A_{i}$, is removed from $A_{i}$ and we apply the dual Somigliana identity to the double connected domain $A^{\prime}=A_{i} \backslash A_{\varepsilon}$. Note that the contour $\mathcal{L}_{\varepsilon}$ of $A_{\varepsilon}$ and the arc $\mathcal{L}_{\varepsilon}^{\prime}$, which is assumed to be the part of the contour $\mathcal{L}_{\varepsilon}$ lying within $A_{i}$, coincide with each other. 
2. If $Q=\stackrel{o}{Q} \in \partial A=\mathcal{L}_{o}$, then the part $A_{i} \cap A_{\varepsilon}$ of the neighborhood $A_{\varepsilon}$ of $Q$ is removed from $A_{i}$ and we apply the dual Somigliana identity to the simply connected region $A^{\prime}=A_{i} \backslash\left(A_{i} \cap A_{\varepsilon}\right)$. In this case, the contour of the simply
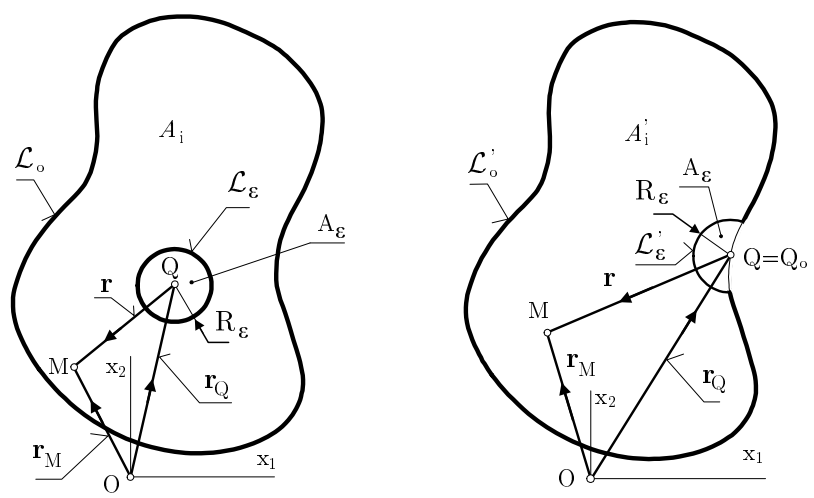

Figure 2 .

connected region just obtained consists of two arcs, the arc $\mathcal{L}_{o}^{\prime}$ left from $\mathcal{L}_{o}$ after the removal of $A_{\varepsilon}$ and the $\operatorname{arc} \mathcal{L}_{\varepsilon}^{\prime}$, i.e., the part of $\mathcal{L}_{\varepsilon}$ that lies within $A_{i}$.

3. If $Q \notin\left(A_{i} \cup \mathcal{L}_{o}\right)$ we apply the dual Somigliana identity to the original region $A_{i}$.

Since both $\stackrel{*}{\mathfrak{u}}_{k}$ and $\mathfrak{u}_{k}$ are elastic states the surface integrals in 4.2 are identically equal to zero.

We now consider each of these three cases, focusing on the main steps of the argument.

1. If $Q \in A_{i}$ it follows from equation 4.2

$$
\begin{aligned}
& \oint_{\mathcal{L}_{o}}\left[\mathfrak{u}_{\lambda}(\stackrel{o}{M}) \stackrel{*}{\mathfrak{t}}_{\lambda}(\stackrel{o}{M})-\stackrel{*}{\mathfrak{u}}_{\lambda}(\stackrel{o}{M}) \mathfrak{t}_{\lambda}(\stackrel{o}{M})\right] \mathrm{d} s_{M}^{o}+\oint_{\mathcal{L}_{\varepsilon}}\left[\mathfrak{u}_{\lambda}(\stackrel{o}{M}) \stackrel{*}{\mathfrak{t}}_{\lambda}(\stackrel{o}{M})-\stackrel{*}{\mathfrak{u}_{\lambda}}(\stackrel{o}{M}) \mathfrak{t}_{\lambda}(\stackrel{o}{M})\right] \mathrm{d} s_{M}^{o} \\
& =e_{k}(Q)\left\{\oint_{\mathcal{L}_{o}}\left[\mathfrak{T}_{k \lambda}(\stackrel{o}{M}, Q) \mathfrak{u}_{\lambda}(\stackrel{o}{M})-\mathfrak{U}_{k \lambda}(\stackrel{o}{M}, Q) \mathfrak{t}_{\lambda}(\stackrel{o}{M})\right] d s_{M}^{\circ}\right. \\
& \left.+\oint_{\mathcal{L}_{\varepsilon}}\left[\mathfrak{T}_{k \lambda}(\stackrel{o}{M}, Q) \mathfrak{u}_{\lambda}(\stackrel{o}{M})-\mathfrak{U}_{k \lambda}(\stackrel{o}{M}, Q) \mathfrak{t}_{\lambda}(\stackrel{o}{M})\right] d s_{M}^{o}\right\}=0 .
\end{aligned}
$$

Since equation 4.3 holds for arbitrary $e_{k}(Q)$ we have

$$
\begin{aligned}
\oint_{\mathcal{L}_{o}}\left[\mathfrak{T}_{k \lambda}(\stackrel{o}{M}, Q)\right. & \left.\mathfrak{u}_{\lambda}(\stackrel{o}{M})-\mathfrak{U}_{k \lambda}(\stackrel{o}{M}, Q) \mathfrak{t}_{\lambda}(\stackrel{o}{M})\right] d s_{M}^{o}+ \\
+ & \oint_{\mathcal{L}_{\varepsilon}}\left[\mathfrak{T}_{k \lambda}(M, Q) \mathfrak{u}_{\lambda}(M)-\mathfrak{U}_{k \lambda}(M, Q) \mathfrak{t}_{\lambda}(M)\right] d s_{M}=0 .
\end{aligned}
$$


To obtain the final form of the above equation, we need to use the integrals detailed below:

- In spite of the singularity of the fundamental solution of order two we can prove that

$$
\lim _{R_{\varepsilon} \rightarrow 0} \oint_{\mathcal{L}_{\varepsilon}} \mathfrak{T}_{\kappa \lambda}(M, Q)\left[\mathfrak{u}_{\lambda}(M)-\mathfrak{u}_{\lambda}(Q)\right] d s_{M}=0 .
$$

- We can also show by performing formal transformations that

$$
\lim _{R_{\varepsilon} \rightarrow 0} \oint_{\mathcal{L}_{\varepsilon}} \mathfrak{U}_{\kappa \lambda}(M, Q) \mathfrak{t}_{\lambda}(M) d s_{M}=0
$$

- It can be proved by relatively long hand calculations that

$$
\lim _{R_{\varepsilon} \rightarrow 0} \oint_{\mathcal{L}_{\varepsilon}} \mathfrak{U}_{3 \lambda}(M, Q) \mathfrak{t}_{\lambda}(M) d s_{M}=\left.\varphi_{3}\right|_{Q}=-\left.\mathfrak{u}_{3}\right|_{Q} .
$$

Making use of the integrals (4.5), 4.6 and 4.7 from equation 4.4 we obtain

$$
\begin{aligned}
\oint_{\mathcal{L}_{o}} & \cdots+\lim _{R_{\varepsilon} \longrightarrow 0} \oint_{\mathcal{L}_{\varepsilon}} \cdots= \\
& =\mathfrak{u}_{k}(Q)-\oint_{\mathcal{L}_{o}} \mathfrak{U}_{k \lambda}(\stackrel{o}{M}, Q) \mathfrak{t}_{\lambda}(\stackrel{o}{M}) d s_{M}^{o}+\oint_{\mathcal{L}_{o}} \mathfrak{T}_{k \lambda}(\stackrel{o}{M}, Q) \mathfrak{u}_{\lambda}(\stackrel{o}{M}) d s_{M}^{o}=0
\end{aligned}
$$

which is, in fact, the first dual Somigliana formula:

$$
\mathfrak{u}_{k}(Q)=\oint_{\mathcal{L}_{o}} \mathfrak{U}_{k \lambda}(\stackrel{o}{M}, Q) \mathfrak{t}_{\lambda}(\stackrel{o}{M}) d s_{M}^{o}-\oint_{\mathcal{L}_{o}} \mathfrak{T}_{k \lambda}(\stackrel{o}{M}, Q) \mathfrak{u}_{\lambda}(\stackrel{o}{M}) d s_{M}^{o} .
$$

2. If $Q=\stackrel{o}{Q}$ our starting point is the formula

$$
\begin{aligned}
\int_{\mathcal{L}_{o}^{\prime}}\left[\mathfrak{T}_{\kappa \lambda}(\stackrel{o}{M}, \stackrel{o}{Q}) \mathfrak{u}_{\lambda}(\stackrel{o}{M})-\mathfrak{U}_{\kappa \lambda}(\stackrel{o}{M} \stackrel{o}{Q}) \mathfrak{t}_{\lambda}(\stackrel{o}{M})\right] d s_{M}^{o}+ \\
\quad+\int_{\mathcal{L}_{\varepsilon}^{\prime}}\left[\mathfrak{T}_{\kappa \lambda}(M, \stackrel{o}{Q}) \mathfrak{u}_{\lambda}(M)-\mathfrak{U}_{\kappa \lambda}(M, \stackrel{o}{Q}) \mathfrak{t}_{\lambda}(M)\right] d s_{M}=0 .
\end{aligned}
$$

Using the limit

$$
\lim _{R_{\varepsilon} \rightarrow 0} \int_{\mathcal{L}_{\varepsilon}^{\prime}} \mathfrak{T}_{\kappa \lambda}(M, \stackrel{o}{Q}) d s_{M}=c_{\kappa \lambda}(\stackrel{o}{Q}),
$$

where $c_{\kappa \lambda}(\stackrel{o}{Q})=\delta_{\kappa \lambda} / 2$, if the contour is smooth at $\stackrel{o}{Q}$ and repeating the line of thought resulting in equation 4.9 , we arrive at the second dual Somigliana formula, i.e. the integral equation of the direct method:

$$
c_{\kappa \lambda}(\stackrel{o}{Q}) \mathfrak{u}_{\lambda}(\stackrel{o}{Q})=\oint_{\mathcal{L}_{o}} \mathfrak{U}_{\kappa \lambda}(\stackrel{o}{M}, \stackrel{o}{Q}) \mathfrak{t}_{\lambda}(\stackrel{o}{M}) d s_{M}^{o}-\oint_{\mathcal{L}_{o}} \mathfrak{T}_{\kappa \lambda}(\stackrel{o}{M}, \stackrel{o}{Q}) \mathfrak{u}_{\lambda}(\stackrel{o}{M}) d s_{M}^{\circ} .
$$

3. If $Q \notin\left(A \cup \mathcal{L}_{o}\right)$ it is not too difficult to check that the third dual Somogliana formula takes the form:

$$
0=\oint_{\mathcal{L}_{o}} \mathfrak{U}_{\kappa \lambda}(\stackrel{o}{M}, Q) \mathfrak{t}_{\lambda}(\stackrel{o}{M}) d s_{M}^{o}-\oint_{\mathcal{L}_{o}} \mathfrak{T}_{\kappa \lambda}(\stackrel{o}{M}, Q) \mathfrak{u}_{\lambda}(\stackrel{o}{M}) d s_{M}^{o} .
$$


Upon substitution of the first dual Somigliana formula 4.9 into the dual kinematic equations 2.1a b) and taking into account that $\mathcal{F}_{\kappa}=\mathfrak{u}_{\kappa}$ we get the stresses $\mathfrak{s}_{m}=$ $\left(t_{11}, t_{12}, t_{22}\right)$ in the form

$$
\mathfrak{s}_{m}(Q)=\oint_{\mathcal{L}_{o}} S_{m \lambda}(\stackrel{o}{M}, Q) \mathfrak{t}_{\lambda}(\stackrel{o}{M}) d s_{\stackrel{o}{o}}-\oint_{\mathcal{L}_{o}} D_{m \lambda}(\stackrel{o}{M}, Q) \mathfrak{u}_{\lambda}(\stackrel{o}{M}) d s_{M}^{o}, \quad Q \in A_{i}
$$

where the matrices of $S_{m \lambda}$ and $D_{m \lambda}$ are

$$
\left[S_{m \lambda}\right]=-2 \mathcal{K} \operatorname{Im} \sum_{k=1}^{2} \frac{d_{k}}{\rho_{k}}\left[\begin{array}{rr}
-\beta_{k}^{3} & \beta_{k}^{2} \\
\beta_{k}^{2} & -\beta_{k} \\
-\beta_{k} & 1
\end{array}\right]
$$

and

$$
\left[D_{m \lambda}\right]=\mathcal{K} \operatorname{Im} \sum_{k=1}^{2} \frac{2 d_{k}}{\rho_{k}^{2}} \times\left[\begin{array}{cc}
D_{11} & D_{12} \\
D_{21} & D_{22} \\
D_{31} & D_{32}
\end{array}\right]
$$

in which

$$
\begin{gathered}
D_{11}=n_{2}\left(-s_{11} \beta_{k}^{4}-s_{12} \beta_{k}^{2}\right)-\frac{n_{1}}{2} s_{66} \beta_{k}^{3}-n_{1}\left[s_{22} \beta_{k}+\left(s_{21}+\frac{s_{66}}{2}\right) \beta_{k}^{3}\right] \\
D_{21}=\frac{n_{2}}{2} s_{66} \beta_{k}^{3}+n_{1}\left(s_{21} \beta_{k}^{4}+s_{22} \beta_{k}^{2}\right)+n_{2}\left[s_{22} \beta_{k}+\left(s_{21}+\frac{s_{66}}{2}\right) \beta_{k}^{3}\right] \\
D_{12}=n_{2}\left(s_{11} \beta_{k}^{3}+s_{12} \beta_{k}\right)+\frac{n_{1}}{2} s_{66} \beta_{k}^{2}-n_{1} \beta_{k}^{2}\left[\left(s_{12}+\frac{s_{66}}{2}\right)+s_{11} \beta_{k}^{2}\right] \\
D_{22}=-\frac{n_{2}}{2} s_{66} \beta_{k}^{2}-n_{1}\left(s_{21} \beta_{k}^{3}+s_{22} \beta_{k}\right)-n_{2} \beta_{k}^{2}\left[\left(s_{12}+\frac{s_{66}}{2}\right)+s_{11} \beta_{k}^{2}\right] \\
D_{13}=-n_{2}\left(s_{11} \beta_{k}^{2}+s_{12}\right)-\frac{n_{1}}{2} s_{66} \beta_{k}+n_{1} \beta_{k}\left[\left(s_{12}+\frac{s_{66}}{2}\right)+s_{11} \beta_{k}^{2}\right] \\
D_{23}=\frac{n_{2}}{2} s_{66} \beta_{k}+n_{1}\left(s_{21} \beta_{k}^{2}+s_{22}\right)+n_{2} \beta_{k}\left[\left(s_{12}+\frac{s_{66}}{2}\right)+s_{11} \beta_{k}^{2}\right]
\end{gathered}
$$

\section{Dual Somigliana formulae for EXterior Regions}

Figure 3 depicts a triple connected region $A_{e}^{\prime}$ bounded by the contours $\mathcal{L}_{o}, \mathcal{L}_{\varepsilon}$ and the circle $\mathcal{L}_{R}$ with radius ${ }_{e} R$ and center $O$. Here $\mathcal{L}_{\varepsilon}$ is the contour of the neighborhood $A_{\varepsilon}$ of $Q$ with radius $R_{\varepsilon}$ while ${ }_{e} R$ is sufficiently large so that the region bounded by $\mathcal{L}_{R}$ covers both $\mathcal{L}_{0}$, and $\mathcal{L}_{\varepsilon}$. If ${ }_{e} R \rightarrow \infty$ and $R_{\varepsilon} \rightarrow 0$ then clearly $A_{e}^{\prime} \rightarrow A_{e}$. 


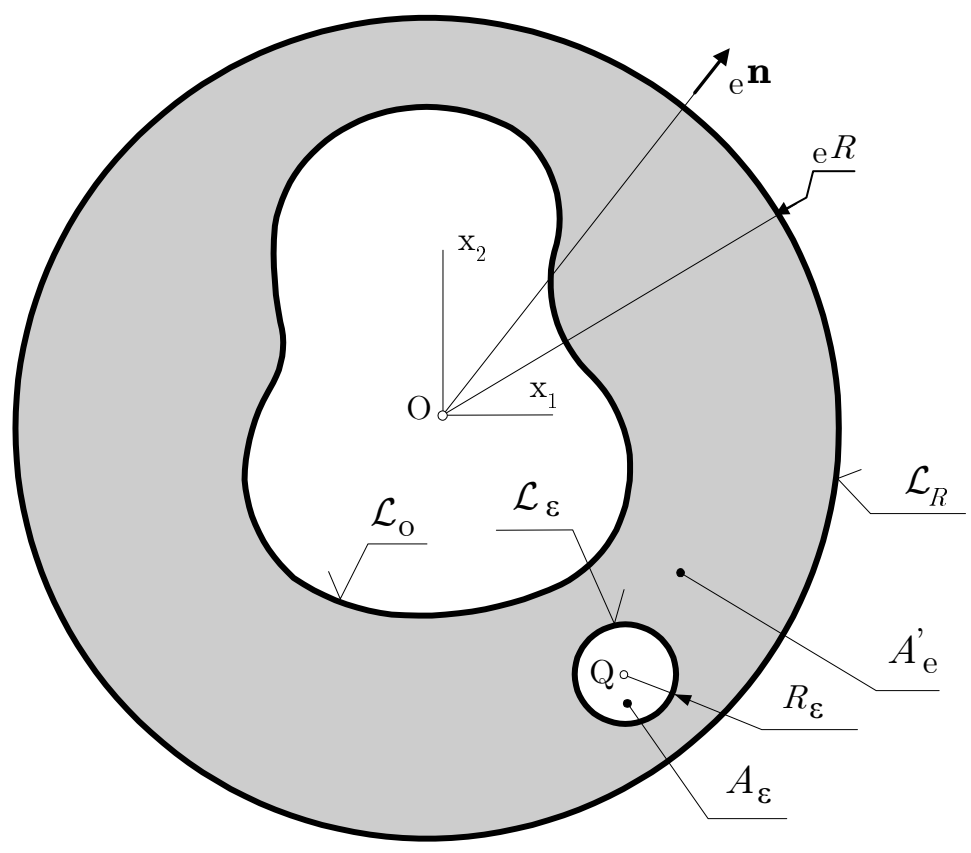

Figure 3.

We shall make the following assumptions:

1. The stresses are constant at infinity. Their values are denoted by

$$
t_{11}(\infty), t_{12}(\infty)=t_{21}(\infty) \text { and } t_{22}(\infty)
$$

2. The rigid body rotation vanishes at infinity:

$$
\varphi_{3}(\infty)=0 .
$$

Let $\mathfrak{u}_{l}(M)$ and $\mathfrak{\mathfrak { u }}_{l}(M)$ be sufficiently smooth elastic states (dual displacements and rotation) on $A_{e}$. The corresponding dual stresses on the contour are denoted by

$$
\mathfrak{t}_{\lambda} \quad \text { and } \quad \stackrel{*}{t}_{\lambda}
$$

respectively. The equation

$$
\begin{aligned}
& \int_{A_{e}^{\prime}}\left[\mathfrak{u}_{r}(M)\left({\stackrel{D}{\mathfrak{D}_{r l}}}^{M} \mathfrak{u}_{l}(M)\right)-\stackrel{\mathfrak{u}}{r}^{*}(M)\left(\stackrel{\mathcal{D}}{r l}^{M} \mathfrak{u}_{l}(M)\right)\right] \mathrm{d} A_{M}= \\
& =\oint_{\mathcal{L}_{o}}\left[\mathfrak{u}_{\lambda}(\stackrel{o}{M}) \stackrel{*}{\mathfrak{t}}_{\lambda}(\stackrel{o}{M})-\stackrel{*}{\mathfrak{u}_{\lambda}}(\stackrel{o}{M}) \mathfrak{t}_{\lambda}(\stackrel{o}{M})\right] \mathrm{d} s_{M}^{o}+\oint_{\mathcal{L}_{\varepsilon}}\left[\mathfrak{u}_{\lambda}(\stackrel{o}{M}) \stackrel{*}{\mathfrak{t}_{\lambda}}(\stackrel{o}{M})-\stackrel{*}{\mathfrak{u}_{\lambda}}(\stackrel{o}{M}) \mathfrak{t}_{\lambda}(\stackrel{o}{M})\right] \mathrm{d} s_{M}^{o} \\
& +\oint_{\mathcal{L}_{R}}\left[\mathfrak{u}_{\lambda}(\stackrel{o}{M}) \stackrel{*}{\mathfrak{t}_{\lambda}}(\stackrel{o}{M})-\stackrel{*}{\mathfrak{u}_{\lambda}}(\stackrel{o}{M}) \mathfrak{t}_{\lambda}(\stackrel{o}{M})\right] \mathrm{d} s_{M}^{\stackrel{o}{M}}
\end{aligned}
$$


is the dual Somigliana identity 4.2 when it is applied to the triple connected region $A_{e}^{\prime}$. Observe that $M$ over a letter denotes that the corresponding derivatives are taken with respect to the coordinates of $M$.

Let again ${ }_{\mathfrak{u}}^{*}(Q)=e_{k}(Q) \mathfrak{U}_{k \kappa}(M, Q)$, which is a non-singular elastic state of the plane in $A_{e}^{\prime}$. We regard $\mathfrak{u}_{l}(M)$ as a different elastic state in the region $A_{e}$. Further we assume that $\mathfrak{u}_{l}(M)$ has the far field pattern (asymptotic behavior)

$$
\begin{gathered}
\mathfrak{u}_{\lambda}(M)=\tilde{\mathfrak{u}}_{\lambda}(M)=c_{\lambda}(\infty)+\varepsilon_{\kappa \rho 3} x_{\rho} t_{\lambda \kappa}(\infty), \\
\mathfrak{u}_{3}(M)=\tilde{\mathfrak{u}}_{3}(M)=-\varphi_{3}(\infty)=0 .
\end{gathered}
$$

when $x_{\beta}$ or equivalently $M$ tends to infinity. Here $c_{\lambda}(\infty)$ is an arbitrary constant which can be set to zero.

Substituting the above quantities into the Somigliana identity $(5.3)$ and taking into account that the surface integrals vanish we have

$$
\begin{aligned}
e_{k}(Q)\left\{\oint_{\mathcal{L}_{o}}\left[\mathfrak{T}_{k \lambda}(\stackrel{o}{M}, Q) \mathfrak{u}_{\lambda}(\stackrel{o}{M})-\mathfrak{U}_{k \lambda}(\stackrel{o}{M}, Q) \mathfrak{t}_{\lambda}(\stackrel{o}{M})\right] d s_{M}+\right. \\
\quad+\oint_{\mathcal{L}_{\varepsilon}}\left[\mathfrak{T}_{k \lambda}(\stackrel{o}{M}, Q) \mathfrak{u}_{\lambda}(\stackrel{o}{M})-\mathfrak{U}_{k \lambda}(\stackrel{o}{M}, Q) \mathfrak{t}_{\lambda}(\stackrel{o}{M})\right] d s_{{ }_{M}}+ \\
\left.\quad+\oint_{\mathcal{L}_{R}}\left[\mathfrak{T}_{k \lambda}(\stackrel{o}{M}, Q) \mathfrak{u}_{\lambda}(\stackrel{o}{M})-\mathfrak{U}_{k \lambda}(\stackrel{o}{M}, Q) \mathfrak{t}_{\lambda}(\stackrel{o}{M})\right] d s_{M}^{o}\right\}=0
\end{aligned}
$$

since

$$
\left.{\stackrel{*}{\mathfrak{t}_{\kappa}}}_{(} \stackrel{o}{M}\right)=e_{l}(Q) \mathcal{T}_{l \kappa}(\stackrel{o}{M}, Q) \text {. }
$$

It is clear that one can omit $e_{k}(Q)$. Recalling the limit 4.8) we get

$$
\begin{aligned}
\mathfrak{u}_{k}(Q)=\lim _{e} \oint_{\mathcal{L}_{R}}[ & {\left[\mathfrak{T}_{k \lambda}(\stackrel{o}{M}, Q) \mathfrak{u}_{\lambda}(\stackrel{o}{M})-\mathfrak{U}_{k \lambda}(\stackrel{o}{M}, Q) \mathfrak{t}_{\lambda}(\stackrel{o}{M})\right] d s_{M}+} \\
& +\oint_{\mathcal{L}_{o}}\left[\mathfrak{T}_{k \lambda}(\stackrel{o}{M}, Q) \mathfrak{u}_{\lambda}(\stackrel{o}{M})-\mathfrak{U}_{k \lambda}(\stackrel{o}{M}, Q) \mathfrak{t}_{\lambda}(\stackrel{o}{M})\right] d s_{M}^{o} .
\end{aligned}
$$

In order to establish the first dual Somigliana formula for the exterior region $A_{e}$ we need to find the limit of the first integral on the right hand side.

In the following, our main objective is to prove that

$$
I_{k}=\lim _{e} \oint_{\mathcal{L}_{R}}\left[\mathfrak{T}_{k \lambda}(\stackrel{o}{M}, Q) \mathfrak{u}_{\lambda}(\stackrel{o}{M})-\mathfrak{U}_{k \lambda}(\stackrel{o}{M}, Q) \mathfrak{t}_{\lambda}(\stackrel{o}{M})\right] d s_{M}^{o}=\tilde{\mathfrak{u}}_{k}(Q) .
$$

The proof uses the first dual SomigLiana formula valid for inner regions and requires simple tools only.

Let us consider the simple connected region $A_{R}$ bounded by the circle $\mathcal{L}_{R}$ with radius ${ }_{e} R$ and center at $O$. We shall assume that the point $Q$ is an inner one.

It is clear that the dual displacements $\tilde{\mathfrak{u}}_{\kappa}(M)$ and rotation field $\tilde{\mathfrak{u}}_{3}(M)$ defined by equations $5.4 \mathrm{a}, \mathrm{b})$ are an elastic state of $A_{R}$ with no body forces. The corresponding dual stresses on the contour are denoted by $\tilde{\mathfrak{t}}_{\kappa}(\stackrel{o}{M})$. It is also obvious that for any 
elastic state of the region $A_{R}$ one can apply the first dual SomigLianA formula. Since $\tilde{\mathfrak{u}}_{k}(M)$ is an elastic state of the orthotropic inner region $A_{R}$ we have

$$
\tilde{\mathfrak{u}}_{k}(Q)=\oint_{\mathcal{L}_{R}}\left[\mathfrak{T}_{k \lambda}(\stackrel{o}{M}, Q) \tilde{\mathfrak{u}}_{\lambda}(\stackrel{o}{M})-\mathfrak{U}_{k \lambda}(\stackrel{o}{M}, Q) \tilde{\mathfrak{t}}_{\lambda}(\stackrel{o}{M})\right] d s_{M}^{o} .
$$

If in addition we take into account the limits

$$
\lim _{e} \mathfrak{u}_{\lambda}(\stackrel{o}{M})=\tilde{\mathfrak{u}}_{\lambda}(\stackrel{o}{M}) \quad \text { and } \quad \lim _{e} \mathfrak{t}_{\lambda}(\stackrel{o}{M})=\tilde{t}_{\lambda}(\stackrel{o}{M})
$$

then we find that the limit of the integral in question in equation $(5.7)$ is really

$$
\lim _{e} \oint_{\mathcal{L}_{R}}\left[\mathfrak{T}_{k \lambda}(\stackrel{o}{M}, Q) \mathfrak{u}_{\lambda}(\stackrel{o}{M})-\mathfrak{U}_{k \lambda}(\stackrel{o}{M}, Q) \mathfrak{t}_{\lambda}(\stackrel{o}{M})\right] d s_{M}^{o}=\tilde{\mathfrak{u}}_{k}(Q)
$$

Consequently, the first dual SomiglianA formula - modified to include a constant stress state at infinity - immediately follows from equations (5.6) and (5.7):

$$
\mathfrak{u}_{k}(Q)=\tilde{\mathfrak{u}}_{k}(Q)+\oint_{\mathcal{L}_{o}} \mathfrak{U}_{k \lambda}(\stackrel{o}{M}, Q) \mathfrak{t}_{\lambda}(\stackrel{o}{M}) d s_{M}^{o}-\oint_{\mathcal{L}_{o}} \mathfrak{T}_{k \lambda}(\stackrel{o}{M}, Q) \mathfrak{u}_{\lambda}(\stackrel{o}{M}) d s_{\stackrel{o}{o}} .
$$

If $Q=\stackrel{o}{M}$ is on $\mathcal{L}_{o}$, nothing changes concerning the limit of the integral taken on $\mathcal{L}_{R}$. Consequently

$$
c_{\kappa \lambda}(\stackrel{o}{Q}) \mathfrak{u}_{\lambda}(\stackrel{o}{Q})=\tilde{\mathfrak{u}}_{k}(\stackrel{o}{Q})+\oint_{\mathcal{L}_{o}} \mathfrak{U}_{\kappa \lambda}(\stackrel{o}{M}, \stackrel{o}{Q}) \mathfrak{t}_{\lambda}(\stackrel{o}{M}) d s_{M}-\oint_{\mathcal{L}_{o}} \mathfrak{T}_{\kappa \lambda}(\stackrel{o}{M}, \stackrel{o}{Q}) \mathfrak{u}_{\lambda}(\stackrel{o}{M}) d s_{M}^{o} .
$$

where $c_{\kappa \rho}=\delta_{\kappa \rho} / 2$ if the contour is smooth at $\stackrel{o}{Q}$. This integral equation is that of the direct method (or the second dual Somigliana formula) for exterior regions.

If $Q$ is inside the contour $\mathcal{L}_{o}$, i,e., in the region $A_{i}$, then it is easy to show that

$$
\left.0=\tilde{\mathfrak{u}}_{k}(Q)+\oint_{\mathcal{L}_{o}} \mathfrak{U}_{k \lambda} \stackrel{o}{M}, Q\right) \mathfrak{t}_{\lambda}(\stackrel{o}{M}) d s_{\stackrel{o}{o}}-\oint_{\mathcal{L}_{o}} \mathfrak{T}_{k \lambda}(\stackrel{o}{M}, Q) \mathfrak{u}_{\lambda}(\stackrel{o}{M}) d s_{M}^{o}
$$

which is the third Somigliana formula for exterior regions.

Recalling formula 4.14 for the stresses, it is easy to check that

$$
\mathfrak{s}_{m}(Q)=\mathfrak{s}_{m}(\infty)+\oint_{\mathcal{L}_{o}} S_{m \lambda}(\stackrel{o}{M}, Q) \mathfrak{t}_{\lambda}(\stackrel{o}{M}) d s_{M}^{o}-\oint_{\mathcal{L}_{o}} D_{m \lambda}(\stackrel{o}{M}, Q) \mathfrak{u}_{\lambda}(\stackrel{o}{M}) d s_{M}^{o} . \quad Q \in A_{e}
$$


6. Calculations of the Stresses on the Boundary

After solving the integral equations of the direct method (equation 4.12 for inner regions, equation (5.12) for exterior regions) we know the dual displacement vector $\mathfrak{u}_{\kappa}$ (the stress functions) and the dual stress vector $\mathfrak{t}_{\kappa}$ (displacement derivatives with respect to the arc coordinate $s$ ) on the contour. The next question is how to determine the stresses on the contour in terms of these quantities.

The calculations leading to the equation system that results in the stresses sought will be carried out in the coordinate system $(x, y)$. In this section we shall not apply indicial no-

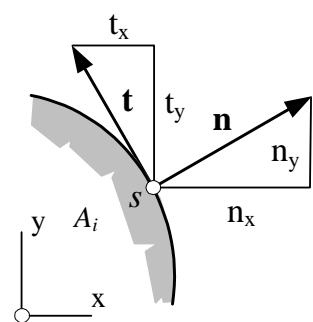

Figure 4. tations including the summation convention.

It is clear from Figure 4 that

$$
\frac{d x}{d s}=t_{x}=-n_{y} \text { and } \frac{d y}{d s}=t_{y}=n_{x}
$$

If we recall formulae $2.1 \mathrm{a}, \mathrm{b})$ which give the stresses in terms of stress functions, we can write (indices 1 and 2 correspond to $x$ and $y$, respectively):

$$
\begin{aligned}
\frac{d \mathcal{F}_{x}}{d s} & =\frac{\partial \mathcal{F}_{x}}{\partial x} \frac{d x}{d s}+\frac{\partial \mathcal{F}_{x}}{\partial y} \frac{d y}{d s}=n_{x} \sigma_{x x}+n_{y} \tau_{x y}, \\
\frac{d \mathcal{F}_{y}}{d s} & =\frac{\partial \mathcal{F}_{y}}{\partial x} \frac{d x}{d s}+\frac{\partial \mathcal{F}_{y}}{\partial y} \frac{d y}{d s}=n_{x} \tau_{x y}+n_{y} \sigma_{y y} .
\end{aligned}
$$

On the basis the definition of the dual stresses $(2.5 \mathrm{~b})$ we have

$$
\begin{aligned}
& -\mathfrak{t}_{x}=\frac{\mathrm{d} u_{x}}{d s}=\frac{\mathrm{d} u_{x}}{d x} \frac{d x}{d s}+\frac{\mathrm{d} u_{x}}{d y} \frac{d y}{d s}=e_{x x} \frac{d x}{d s}+\frac{\mathrm{d} u_{x}}{d y} \frac{d y}{d s} \\
& -\mathfrak{t}_{y}=\frac{\mathrm{d} u_{y}}{d s}=\frac{\mathrm{d} u_{y}}{d x} \frac{d x}{d s}+\frac{\mathrm{d} u_{y}}{d y} \frac{d y}{d s}=\frac{\mathrm{d} u_{y}}{d x} \frac{d x}{d s}+e_{y y} \frac{d y}{d s} .
\end{aligned}
$$

Multiplying by $t_{x}=-n_{y}$ and $t_{y}=n_{x}$ throughout and combining the equations obtained from (6.3) and 6.4 we arrive at

$$
n_{y} \mathfrak{t}_{x}-n_{x} \mathfrak{t}_{y}=e_{x x}\left(\frac{d x}{d s}\right)^{2}+e_{y y}\left(\frac{d y}{d s}\right)^{2}+\frac{d y}{d s} \frac{d x}{d s} \overbrace{\left(\frac{\partial u_{x}}{\partial y}+\frac{\partial u_{y}}{\partial x}\right)}^{2 e_{x y}}
$$

from which making use of the Hooke law and the geometrical relations (6.1) we obtain

$$
n_{y} t_{x}-n_{x} t_{y}=\left(n_{y}{ }^{2} s_{11}+n_{x}{ }^{2} s_{21}\right) \sigma_{x}-n_{x} n_{y} \frac{s_{66}}{2} \tau_{x y}+\left(n_{y}{ }^{2} s_{12}+n_{x}{ }^{2} s_{22}\right) \sigma_{y} .
$$

Equations $6.2 \mathrm{a}, \mathrm{b})$ and $(6.6)$ can be arranged in a matrix form 


$$
\begin{aligned}
{\left[\begin{array}{ccc}
n_{x} & n_{y} & 0 \\
0 & n_{x} & n_{y} \\
n_{y}^{2} s_{11}+n_{x}^{2} s_{21} & -n_{x} n_{y} \frac{s_{66}}{2} & n_{y}^{2} s_{12}+n_{x}^{2} s_{22}
\end{array}\right]\left[\begin{array}{c}
\sigma_{x x} \\
\tau_{x y} \\
\sigma_{y y}
\end{array}\right]=} & =\left[\begin{array}{c}
d \mathcal{F}_{x} / d s \\
d \mathcal{F}_{y} / d s \\
n_{y} \mathfrak{t}_{x}-n_{x} \mathfrak{t}_{y}
\end{array}\right] .
\end{aligned}
$$

Since the outer normal $\mathbf{n}$, the stress functions $\mathcal{F}_{x}, \mathcal{F}_{y}$ and the dual stresses $\mathfrak{t}_{x}, \mathfrak{t}_{y}$ are all known on the contour as soon as we have solved the integral equation of the direct method, solution of the above linear equations will result in the stress components sought.

\section{EXAmples}

A program has been written in Fortran 90 in order to solve the integral equations 4.12 and 5.12 of the direct method on inner and exterior regions numerically. We have applied quadratic boundary elements. Let $n_{b e}$ and $n_{b n}$ be the number of boundary elements and that of the boundary nodes. Further let

$$
\mathbf{u}_{j}=\left[\begin{array}{c}
\mathfrak{u}_{1}^{j} \\
\mathfrak{u}_{2}^{j}
\end{array}\right] \quad \text { and } \quad \mathbf{t}_{j}=\left[\begin{array}{c}
\mathfrak{t}_{1}^{j} \\
\mathfrak{t}_{2}^{j}
\end{array}\right] \quad j=1, \ldots, n_{b n}
$$

be the matrices of the dual displacements and dual stresses at node $j$ on the boundary.

Following the well-known procedure valid for the primal formulation - see for instance [9] - solution of the dual integral equation 4.12 can be reduced to the solution of the linear equations

$$
\begin{aligned}
& {\left[\begin{array}{cccc}
\mathbf{h}_{11} & \mathbf{h}_{12} & \cdots & \mathbf{h}_{1 n_{b n}} \\
\mathbf{h}_{21} & \mathbf{h}_{22} & \cdots & \mathbf{h}_{2 n_{b n}} \\
\ldots \ldots \ldots \ldots \ldots \ldots \ldots \ldots & \ldots \ldots \ldots \ldots \\
\mathbf{h}_{n_{b n} 1} & \mathbf{h}_{n_{b n} 2} & \cdots & \mathbf{h}_{n_{b n} n_{b n}}
\end{array}\right]\left[\begin{array}{l}
\mathbf{u}_{1} \\
\mathbf{u}_{2} \\
\cdots \\
\mathbf{u}_{n_{b n}}
\end{array}\right]=} \\
& =\left[\begin{array}{cccc}
\mathbf{b}_{11} & \mathbf{b}_{12} & \cdots & \mathbf{b}_{1 n_{b n}} \\
\mathbf{b}_{21} & \mathbf{b}_{22} & \cdots & \mathbf{b}_{2 n_{b n}} \\
\ldots \ldots \ldots \ldots \ldots \ldots \ldots & \ldots \ldots \ldots \ldots \\
\mathbf{b}_{n_{b n} 1} & \mathbf{b}_{n_{b n} 2} & \cdots & \mathbf{b}_{n_{b n} n_{b n}}
\end{array}\right]\left[\begin{array}{l}
\mathbf{t}_{1} \\
\mathbf{t}_{2} \\
\cdots \\
\mathbf{t}_{n_{b n}}
\end{array}\right]
\end{aligned}
$$

where the $2 \times 2$ submatrices $\mathbf{h}_{i j}$ and $\mathbf{b}_{i j}$ are computed from the integrals

$$
\begin{gathered}
\hat{\mathbf{h}}_{i j}=\left[\sum_{e \in j} \int_{\mathcal{L}_{e}} \mathfrak{T}_{\kappa \lambda}\left(Q_{i}, \eta\right) N^{a(j, e)}(\eta) J(\eta) d \eta\right], \quad \mathbf{h}_{i j}= \begin{cases}\hat{\mathbf{h}}_{i i}+\mathbf{c}_{i i}, & \text { if } i=j \\
\hat{\mathbf{h}}_{i j}, & \text { if } i \neq j\end{cases} \\
\mathbf{b}_{i j}=\left[\sum_{e \in j} \int_{\mathcal{L}_{e}} \mathfrak{U}_{\kappa \lambda}\left(Q_{i}, \eta\right) N^{a(j, e)}(\eta) J(\eta) d \eta\right]
\end{gathered}
$$

in which (a) the summation is to be carried out for those boundary elements having the nodal point $j$ as their common nodal point; (b) $i$ identifies the fixed nodal point 
$Q_{i}$ referred to as the collocation point; (c) $N^{a(j, e)}(\eta)$ is the a-th shape function for which $a(j, e)$ is the local number of the global nodal point $j$ on element $e$; (d) $\mathbf{c}_{i i}$ is the matrix of $c_{\kappa \lambda}\left(Q_{i}\right)$ in equation 4.11; (e) $J(\eta)$ is the Jacobian. Equation system (7.2) can be rewritten as

$$
\mathbf{H u}=\mathbf{B t}
$$

We remark that determination of the diagonal elements $\mathbf{h}_{i i}, i=1, \ldots, n_{b n}$ requires the computation of strongly singular integrals.

If the region under consideration is an exterior one then in accordance with integral equation 5.12 the right-hand side of equation $(7.5)$ is to be supplemented by the term

$$
\tilde{\mathbf{u}}^{T}=[\underbrace{\tilde{\mathfrak{u}}_{1}^{1} \tilde{\mathfrak{u}}_{2}^{1}}_{\tilde{\mathbf{u}}_{1}^{T}}|\underbrace{\tilde{\mathfrak{u}}_{1}^{2} \tilde{\mathfrak{u}}_{2}^{2}}_{\tilde{\mathbf{u}}_{2}^{T}}| \cdots \mid \underbrace{\tilde{\mathfrak{u}}_{1}^{n_{b n}} \tilde{\mathfrak{u}}_{2}^{n_{b n}}}_{\tilde{\mathbf{u}}_{n_{b n}}^{T}}]
$$

and takes the form

$$
\mathbf{H u}=\tilde{\mathbf{u}}+\mathbf{B t}
$$

If the dual displacements (the stress functions) are constant the stresses and the strains vanish. If in addition we assume that the rotaion $\varphi^{3}$ vanishes - this does not violate generality - then the dual stresses also vanish. Consequently

$$
\sum_{j=1}^{2 n_{b n}} H_{i j}=0 \quad \text { from where } \quad H_{i i}=-\sum_{\substack{j=1 \\(i \neq j)}}^{2 n_{b n}} H_{i j} \quad i=1,2, \ldots, 2 n_{b n}
$$

where $H_{i j}$ is an element of the matrix $\mathbf{H}$. Making use of the equation above we can avoid the computation of strongly singular integrals for interior boundary value problems.

For an exterior region, the strongly singular integrals can be determined using an equation similar to equation $(7.8)$, which we present here without proof:

$$
H_{i i}=-\sum_{\substack{j=1 \\(i \neq j)}}^{2 n_{b n}} H_{i j}+1 \quad i=1,2, \ldots, 2 n_{b n}
$$

We have solved one simple internal test problem and two external boundary value problems. First we consider a beam in pure bending (Figure 5), second the coordinate plane with a circular hole (Figure 6p.); third the coordinate plane with a rigid inclusion (Figure 6.). The material is birch for which $s_{11}=8.497 \times 10^{-5}, s_{12}=s_{21}=$ $-6.11 \times 10^{-2}, s_{22}=1.6999 \times 10^{-4}$ and $s_{66}=1.456 \times 10^{-3}\left[\mathrm{~mm}^{2} / \mathrm{N}\right]$. 


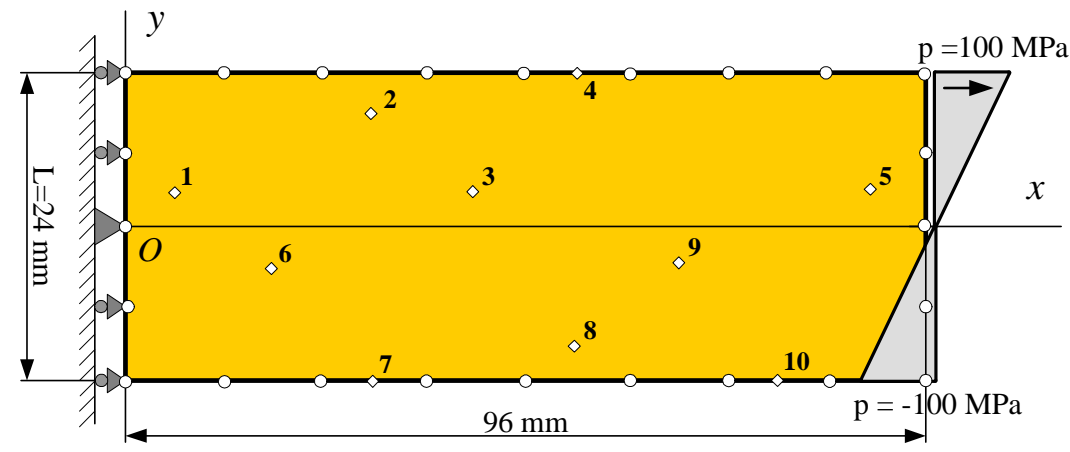

Figure 5.

The first problem has a closed form solution for the stresses:

$$
\sigma_{x x}=200 y / L, \quad \sigma_{y y}=0 ;, \quad \tau_{x y}=0 .
$$

The contour is divided into twenty elements of equal length as shown in Figure 6 . Table 1 presents the computed results which are in close agreement with the accurate values.

Table 1.. Results for the pure bending of a beam

\begin{tabular}{|c|r|r|r|}
\hline \multicolumn{3}{|c|}{ Pure bending: stresses at the inner - and contour points } \\
\hline The point & \multicolumn{3}{|c|}{ Stresses $[\mathrm{MPa}]$} \\
\cline { 2 - 4 } Selected & \multicolumn{1}{|c|}{$\sigma_{x x}$} & \multicolumn{1}{|c|}{$\tau_{x y}$} & $\sigma_{y y}$ \\
\hline $1(6 ; 3)$ & 25.00023 & 0.000707695 & -0.000186628 \\
\hline $2(30 ; 9)$ & 74.99414 & 0.000184937 & -0.000164188 \\
\hline $3(42 ; 3)$ & 24.99820 & -0.000128817 & -0.000083552 \\
\hline $4(54 ; 12)$ & 100.02202 & 0.000000000 & 0.000000000 \\
\hline $5(90 ; 3)$ & 25.00023 & 0.000707695 & -0.000186628 \\
\hline $6(18 ;-3)$ & -24.99898 & -0.000708675 & 0.000120160 \\
\hline $7(30 ;-12)$ & -100.02220 & 0.000000000 & 0.000000000 \\
\hline $8(54 ;-9)$ & -74.99372 & -0.000055471 & 0.000156285 \\
\hline $9(66 ;-3)$ & -24.99844 & 0.000398726 & 0.000092128 \\
\hline $10(78 ;-12)$ & -100.02223 & 0.000000000 & 0.000000000 \\
\hline
\end{tabular}

For the exterior boundary value problems we shall assume that

$$
\tau_{x y}(\infty)=\sigma_{y y}(\infty)=0 \quad \text { and } \quad \sigma_{x x}(\infty)=p=\text { constant }
$$

For completeness Figure 6a shows the region to use if we solve the integral equation of the direct method in its traditional form - see equation 4.9 - I.e. if the exterior region is replaced by a bounded one. 


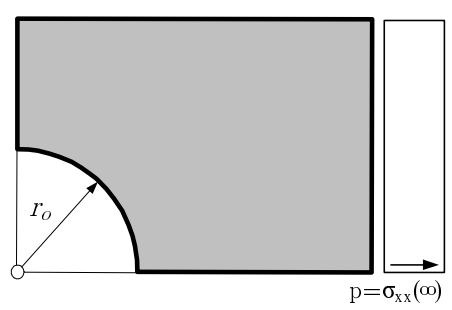

(a)
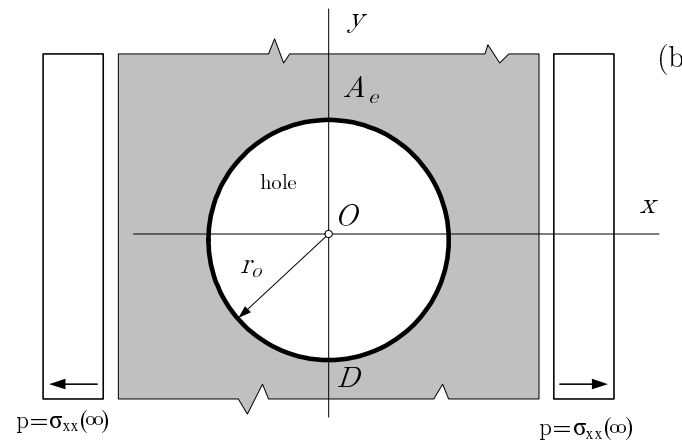

(b)
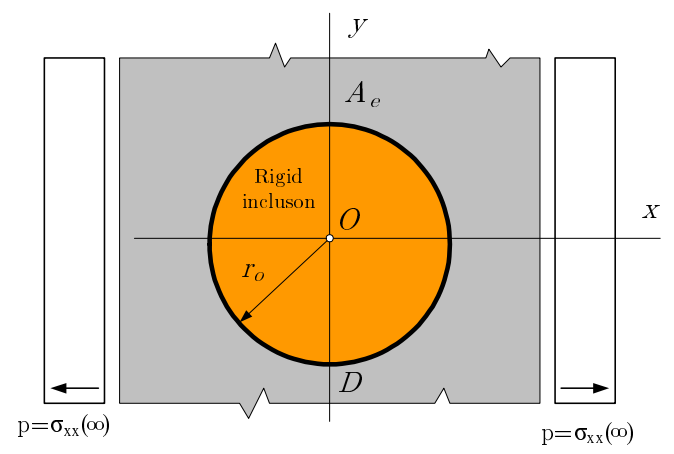

(c)

Figure 6 .

LEKHTNISKI's book 15 contains closed form solutions for the stresses on the boundary, as well as numerical values which can be found in Table 17 on page 197 . In this paper we show the results as computed by solving the integral equation and the results taken from [15] - see Tables 2 and 3 . We used a polar coordinate system, and the tables contain the quotients $\sigma_{\theta} / p$ for the plane with circular hole and $\sigma_{r} / p$, $\tau_{r \theta} / p$ and $\sigma_{\theta} / p$ for the plane with the rigid circular inclusion.

Table 2.. Results for the circular hole

\begin{tabular}{|c|rr|}
\hline \multirow{3}{*}{ Polar angle } & \multicolumn{2}{|c|}{ Circular hole } \\
\cline { 2 - 3 } & \multicolumn{2}{|c|}{$\sigma_{\theta} / p$} \\
Lekhnitski \\
& & {$[15]$} \\
\hline $0^{\circ}$ & -0.70744 & -0.707 \\
$15^{\circ}$ & -0.33928 & -0.340 \\
$30^{\circ}$ & 0.06951 & 0.069 \\
$45^{\circ}$ & 0.40451 & 0.404 \\
$60^{\circ}$ & 0.96605 & 0.966 \\
$75^{\circ}$ & 2.57736 & 2.577 \\
$90^{\circ}$ & 5.45409 & 5.453 \\
\hline
\end{tabular}


Table 3. Results for the rigid inclusion

\begin{tabular}{|c|c|c|c|c|c|c|}
\hline \multirow[b]{2}{*}{ Polar angle } & \multicolumn{6}{|c|}{ Rigid kernel } \\
\hline & \multicolumn{2}{|c|}{$\begin{array}{c}\sigma_{r} / p \\
\text { Lekhnitski } \\
{[15}\end{array}$} & \multicolumn{2}{|c|}{$\begin{array}{c}\tau_{r \theta} / p \\
\text { Lekhnitski } \\
15\end{array}$} & \multicolumn{2}{|c|}{$\begin{array}{c}\sigma_{\theta} / p \\
\text { Lekhnitski } \\
15\end{array}$} \\
\hline $0^{\circ}$ & 1.2363 & 1.237 & 0.0000 & 0.000 & 0.0444 & 0.044 \\
\hline $15^{\circ}$ & 1.1558 & 1.156 & -0.2999 & -0.299 & 0.0936 & 0.093 \\
\hline $30^{\circ}$ & 0.9364 & 0.937 & -0.5188 & -0.519 & 0.2701 & 0.270 \\
\hline $45^{\circ}$ & 0.6370 & $\underline{0.698}$ & -0.5986 & -0.599 & 0.5158 & 0.516 \\
\hline $60^{\circ}$ & 0.3377 & 0.338 & -0.5181 & -0.519 & 0.6990 & 0.699 \\
\hline $75^{\circ}$ & 0.1188 & 0.119 & -0.2987 & -0.299 & 0.5627 & 0.564 \\
\hline $90^{\circ}$ & 0.0389 & 0.039 & 0.0000 & 0.000 & 0.0028 & 0.003 \\
\hline
\end{tabular}

We remark that the underlined value is mistaken in book [15].

\section{Concluding Remarks}

The present paper has dealt with the following issues:

1. We have presented the equations of plane elasticity for an orthotropic body in terms of stress functions of order one. We have also clarified what are the supplementary conditions of single valuedness for a class of mixed boundary value problems in the dual system of plane elasticity.

2. By applying Galorkin functions and following the procedure presented among others in book [14 by KUPRADZE we have derived the dual fundamental solutions of order one and two for plane problems of orthotropic bodies.

3. We have set up the dual Somigliana relations both for inner regions and for exterior ones. A constant stress state at infinity is a part of the formulation we have developed for exterior regions. The integral representation of the stresses has also been established.

4. Three simple boundary value problems (one for an interior region, the other two for the same exterior region) have been solved numerically in order to demonstrate the applicability of the solution algorithm.

We remark that the supplementary conditions of single valuedness should be incorporated into the algorithm if (a) the number of arcs on which tractions are prescribed is more than one or (b) if in addition to this the region under consideration is multiply connected. Work on these issues is in progress.

\section{REFERENCES}

1. Tonti, E.: A mathematical model for physical theories I. II. Rendiconti Accademia Nazionale dei Lincei, pp. 175-181; 351-356.

2. Rizzo, F. J. and Shippy, D. J.: A method for stress determination in plane anisotropic bodies. Journal of Composite Materials, 4(1), (1970), 36-61.

3. Rizzo, F. J.: An integral equation approach to boundary value problems of classical elastostatics. Q. J. Appl. Math., 25, (1967), 83-95. 
4. Vable, M. and Sikarskie, D. L.: Stress analysis in plane orthotropic material by the boundary element method. Int. J. Solids Structures, 24(1), (1988), 1-11.

5. Shiah, Y. C. and TAN, C. L.: BEM treatment of two-dimensional anisotropic field problems by direct domain mapping. Engineering Analysis with Boundary Elements, 20, (1997), 347-351.

6. Shiah, Y. C. and TAN, C. L.: BEM treatment of three-dimensional anisotropic field problems by direct domain mapping. Engineering Analysis with Boundary Elements, 28, (2004), 43-52.

7. Huang, L., Sun, X., Liu, Y., and Cen, Z.: Parameter identification for twodimensional orthotropic material bodies by the boundary element method. Engineering Analysis with Boundary Elements, 28(2), (2004), 109-121.

8. Banarjee, P. K. and Butterfield, R.: Boundary Element Methods in Engineering Science. Mir, Moscow, 1984.

9. BanarJee, P. K.: The Boundary Element Methods in Engineering. McGraw-Hill, New York, 1994.

10. SzeIdL, G.: Kinematic admissibility of strains for same mixed boundary value problems in the dual system of micropolar theory of elasticity. Journal of Computational and Applied Mechanics, 1(2), (2000), 191-203.

11. Szeidl, G.: Boundary integral equations for plane problems in terms of stress functions of order one. Journal of Computational and Applied Mechanics, 2(2), (2001), 237-261.

12. Szeidl, G. and SzirbiK, S.: New Developments in the Boundary Element Method: Boundary Contour Method for Plane Problems in a Dual Formulation with Quadratic Shape Functions, Chapter 14. Springer-Verlag, 2002.

13. Szeidl, G.: Problems of Continuum Mechanics in a Dual Formulation. Dr. Sc. Thesis, Hungarian Academy of Sciences, 2005. (in Hungarian).

14. В. Д. Купрадзе: Методы потенциала в теории упругости. Физматгиз, Москва, 1963.

15. С. Г. Лехницки: Теория упругости анизотропного тела. Второе Издание, Наука, Москва, 1977. 